\title{
SLM and PTS Method with Clipping for Reducing Complexity of PAPR Reduction in OFDM
}

\author{
Khyati Keyur Desai \\ Lecturer in Electronics \& Communication Engg, Electrical Department, Government Polytechnic, Valsad, India
}

\begin{abstract}
Orthogonal frequency division multiplexing (OFDM) also referred as multicarrier communication systems, have become a key technology in current and for future communication systems. Due to OFDM's immunity to many channel imperfections, it is the ideal modulation scheme for many applications which transmit signals in hostile environments. A major drawback of OFDM is the high peak-to-average-power ratio (PAPR) problem, which can lead to low power efficiency and nonlinear distortion at the transmitter power amplifier. Selected mapping (SLM) and Partial transmit sequences (PTS) are powerful and distortion less peak power reduction schemes for OFDM. Both reduce the PAPR by generating alternative subcarrier vector that are statistically independent OFDM symbols for a given data frame and transmitting the symbol with the lowest peak power. As we increase alternative subcarrier vector, PAPR still reduces but computational complexity also increases. In this paper the technique of clipping is used in conjunction with SLM and PTS to reduce computational complexity.
\end{abstract}

Keywords: Orthogonal frequency division multiplexing (OFDM), Peak-to-Average Power Ratio (PAPR), Clipping Ratio (CR), Selected mapping (SLM), Partial transmit sequences (PTS).

\section{INTRODUCTION}

Orthogonal Frequency Division Multiplexing has many advantages including resistance to multipath fading and high data rates. It has therefore been chosen as the modulation standard for IEEE802.11a/g WLAN, Worldwide Interoperability for Microwave Access (WiMax), Digital Audio Broadcasting (DVB). However a major drawback of OFDM is the manner in which the phases can align in the frequency domain causing high peaks to result in the time domain [1]. High peak values cause saturation of the power amplifier and both in-band and out-of-band distortion when limiting effects occurs. To prevent such phenomena amplifiers are normally "backed off" by approximately the PAPR. This however severely impacts power amplifier efficiency, making it preferable to reduce the PAPR of the signal before it enters the power amplifier [1]. For PAPR reduction number of techniques have been developed e.g. Clipping, Coding, Selected Mapping, Partial Transmit Sequences, Tone Injection, and Tone Reservation. Selected mapping (SLM) \& Partial transmit sequences (PTS) are powerful and distortion less peak power reduction schemes for OFDM. In SLM the transmitter selects one favourable transmit signal from a set of sufficiently different signals which all represent the same information, while in PTS the transmitter constructs its transmit signal with low PAPR by coordinated addition of appropriately phase rotated signal parts[2]. Both are distortion less technique, but require overhead information bits to be sent along with the transmitted signal so that the receiver can reverse the PAPR reduction performance and recover the data. Clipping is a simplest distortion technique for PAPR reduction. It does not require any side information to be sent which mean the data rate is unchanged even after PAPR reduction and have low complexities compared to the distortion less techniques.

\section{PAPR IN OFDM}

OFDM signals have a higher Peak-to-Average Power Ratio (PAPR) than single carrier signals. The reason for this is that in the time domain, a multicarrier signal is the sum of many narrowband signals. At some time instances, this sum is large, at other times it is small, which mean that the peak value of the signal is substantially larger than the average value. This high PAPR is one of the most important implementation challenges that face OFDM because it reduces the efficiency and hence increases the cost of the RF power amplifier. Let $A=\left[\mathrm{A}_{0} \mathrm{~A}_{1} \ldots . . \mathrm{A}_{\mathrm{N}-1}\right]$ denote an input symbol sequence in the frequency domain, where $A_{K}$ represents the complex data of the $k^{\text {th }}$ sub carrier and $N$ the number of sub carriers of OFDM signal. Let $\mathrm{T}$ be a period of input symbol and NT a period of OFDM signal. The OFDM signal is generated by summing all the $\mathrm{N}$ modulated sub carriers each of which is separated by $1 / \mathrm{T}$. Then the complex OFDM signal in time domain is expressed as

$$
a_{t}=\frac{1}{\sqrt{N}} \sum_{n=0}^{N-1} A_{n} e^{i 2 \pi n t / N T}, 0 \leq t \leq N T
$$

Where, $\mathrm{t}$ is continuous time index. The OFDM signal sampled at Nyquist rate can be written as

$$
a_{k}=\frac{1}{\sqrt{N}} \sum_{n=0}^{N-1} A_{n} e^{j 2 \pi k / N}, k=0,1, \ldots, N-1
$$

Which can also be in a vector form, called an OFDM signal sequence, as $a=\left[a_{0} a_{1} \ldots a_{N-1}\right]$. In fact, $a$ correspond to inverse fast Fourier transform (IFFT) of A. The PAPR of OFDM signal sequence $a$ is defined as the ratio between maximum instantaneous power and its average power, which can be written as, 


$$
\operatorname{PAPR}(a)=\frac{\max _{0 \leq k \leq N-1}\left|a_{k}\right|^{2}}{\mathrm{E}\left[\left|a_{k}\right|^{2}\right]}
$$

Where E[.] denotes the expectation operator [3][6]. Statistically it is possible to characterize the PAPR distribution (probability that PAPR exceeds given threshold $\gamma$ ) using its cumulative distribution function (CDF) or complementary cumulative distribution function (CCDF). For the case of OFDM, the following expression for the CCDF holds,

$$
\begin{aligned}
& \mathrm{P}_{r}(P A P R<\gamma)=1-(1-\exp (-\gamma))^{N} \\
& C C D F_{P A P R}=1-C D F_{P A P R}
\end{aligned}
$$

\section{SELECTED MAPPING}

In this most general approach it is assumed that $U$ statistically independent alternative transmits sequences $a_{\mu}{ }^{(u)}$ represent the same information. Then, that sequence $\tilde{a}_{\mu}=a_{\mu}{ }^{\left(\tilde{u}_{\mu}\right)}$ with the lowest PAPR, denoted as $\tilde{\chi}_{\mu}$, is selected for transmission. The probability that $\tilde{\chi}_{\mu}$ exceeds $\chi_{0}$ is approximated by,

$$
P_{r}\left\{\tilde{\chi}_{\mu}>\chi_{0}\right\}=\left(1-\left(1-e^{-\chi_{0}}\right)^{N}\right)^{U}
$$

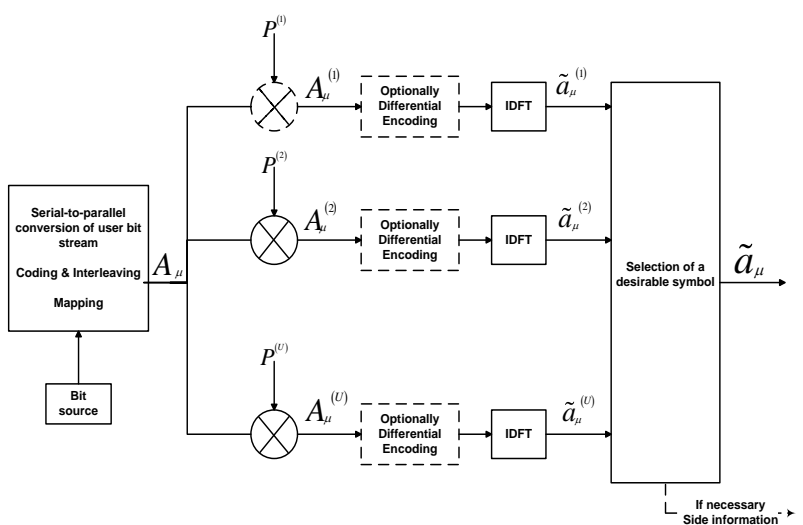

Fig. 1 PAPR reduction in SLM-OFDM [2]

Because of the selected assignment of binary data to the transmit signal, this principle is called selected mapping. A set of $U$ markedly different, distinct, pseudo-random but fixed vectors,

$$
\begin{aligned}
& P^{(u)}=\left[P_{0}^{(u)}, \ldots, P_{D-1}{ }^{(u)}\right] \\
& P_{v}^{(u)}=e^{+j \varphi_{v}{ }^{(u)}}, \varphi_{v}{ }^{(u)} \in[0,2 \pi), 0 \leq v<N, 1 \leq u \leq U,
\end{aligned}
$$

(6)

must be defined. The subcarrier vector $A_{\mu}$ is multiplied subcarrier wise with each one of the $\mathrm{U}$ vectors $P^{(u)}$, resulting in a set of $U$ different subcarrier vectors $A_{\mu}^{(u)}$ with components,

$$
A_{\mu, v}{ }^{(u)}=A_{\mu, v} \cdot P_{v}^{(u)}, \mathrm{O} \leq v<N, 1 \leq u \leq U
$$

Then, all $\mathrm{U}$ alternative subcarrier vectors are transformed into time domain to get $\mathrm{a}_{\mu}{ }^{(u)}=\operatorname{IDFT}\left\{A_{\mu}{ }^{(u)}\right\}$ and finally that transmit sequence $\tilde{a}_{\mu}=a_{\mu}{ }^{\left(\tilde{u}_{\mu}\right)}$ with the lowest PAPR $\tilde{\chi}_{\mu}$ is chosen. The SLM-OFDM transmitter is depicted in Fig. 1 where it is visualized that one of the alternative subcarrier vectors can be the unchanged original one. Optionally, differentially encoded modulation may be applied before the IDFT and right after generating the alternative OFDM symbols. At the receiver, differential demodulation has to be implemented right after the DFT [2][5].

\section{PARTIAL TRANSMIT SEQUENCES}

In this method, the subcarrier vector $A_{\mu}$ is partitioned into $\mathrm{V}$ pair wise disjoint subblocks $A_{\mu}{ }^{(\nu)}, 1 \leq v \leq V$. All subcarrier positions in $A_{\mu}{ }^{(\nu)}$, which are already represented in another subblock are set to zero, so that $A_{\mu}=\sum_{v=1}^{V} A_{\mu}^{(v)}$

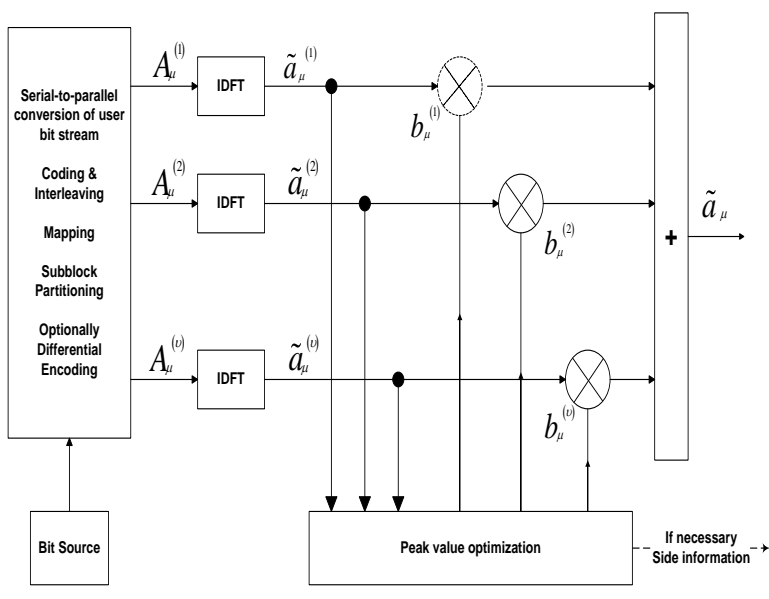

Fig. 2 PAPR reduction in PTS-OFDM [2]

We introduce complex-valued rotation factors $b_{\mu}{ }^{(\nu)}=e^{+j \varphi_{\mu}(\nu)}, \varphi_{\mu}{ }^{(\nu)} \in[0,2 \pi), 1 \leq v \leq V, \forall_{\mu}$ enabling a modified subcarrier vector $\tilde{A}_{\mu}=\sum_{v=1}^{V} b_{\mu}{ }^{(v)} \cdot A_{\mu}{ }^{(v)}$, which represents the same information as $A_{\mu}$, if the set $\left\{b_{\mu}{ }^{(v)}, 1 \leq v \leq V\right\}$ (as side information) is known for each $\mu$. Clearly, simply a joint rotation of all subcarriers in sub block $v$ by the same angle $\varphi_{\mu}{ }^{(v)}=\arg \left(b_{\mu}{ }^{(v)}\right)$ is performed. To calculate $\tilde{a}_{\mu}=\operatorname{IDFT}\left\{\tilde{A}_{\mu}\right\}$, the linearity of the IDFT is exploited. Accordingly, the sub blocks are transformed by $\mathrm{V}$ separate and parallel D-point IDFTs, yielding

$$
\tilde{a}_{\mu}=\sum_{v=1}^{V} b_{\mu}{ }^{(v)} \operatorname{IDFT}\left\{A_{\mu}^{(v)}\right\}=\sum_{v=1}^{V} b_{\mu}^{(v)} \cdot a_{\mu}{ }^{(v)}
$$


Where the $\mathrm{V}$ so-called partial transmit sequences $a_{\mu}{ }^{(\nu)}=\operatorname{IDFT}\left\{A_{\mu}{ }^{(v)}\right\}$ have been introduced. The PTSOFDM transmitter is shown in Fig. 2 with the hint, that one PTS can always be left unrotated [2]. Based on them a peak value optimization is performed by suitably choosing the free parameters $b_{\mu}{ }^{(v)}$ such that the PAPR is minimized for $\tilde{b}_{\mu}^{(v)}$.The $b_{\mu}^{(v)}$ may be chosen with continuousvalued phase angle, but more appropriate in practical systems is a restriction on a finite set of $\mathrm{W}$ (e.g. 4) allowed phase angles. The optimum transmit sequence then is,

$$
\tilde{a}_{\mu}=\sum_{v=1}^{V} \tilde{b}_{\mu}^{(v)} \cdot a_{\mu}^{(v)} \text {. }
$$

Both scheme require, that the receiver has knowledge about the generation of the transmitted OFDM signal in symbol period $\mu$. Thus, in PTS the set with all rotation factors $\tilde{b}_{\mu}{ }^{(v)}$ and in SLM the number $\tilde{u}_{\mu}$ of the selected $P^{\left(\tilde{u}_{\mu}\right)}$ has to be transmitted to the receiver unambiguously so that this one can denote the subcarriers appropriately. The number of bits required for canonical representation of this side information is the redundancy $R_{a p}$ introduced by the PAPR reduction scheme with PTS \& SLM. As this side information is of highest importance to recover the data, it should be carefully protected by channel coding. In PTS the number of admitted combinations of rotation angles $\left\{b_{\mu}{ }^{(\nu)}\right\}$ should not be excessively high, to keep the explicitly transmitted side information within a reasonable limit. If in PTS each $b_{\mu}{ }^{(v)}$ is exclusively chosen from a set of $\mathrm{W}$ admitted angles, then $\mathrm{R}_{\mathrm{ap}}=(\mathrm{V}-1) \log _{2} \mathrm{~W}$ bits per OFDM symbol are needed for this purpose. In SLM Rap = $\log _{2} \mathrm{U}$ bits are required for side information. In PTS the choice $b_{\mu}{ }^{(v)} \in\{ \pm 1, \pm j\}(\mathrm{W}=4)$ is very interesting for an efficient implementation, as actually no multiplication must be performed, when rotating and combining the PTSs $a_{\mu}{ }^{(v)}$ to the peak-optimized transmit sequence $\tilde{a}_{\mu}$ in Eq.9. For SLM, choosing $P_{v}^{(u)}$ from the latter set has the same advantage, when generating the alternative subcarrier vectors by applying Eq.7 [2][4].

\section{CLIPPING}

The clipping technique is used to limit the amplitude of the peaks to a particular clipping level and fix the phase when the peaks are higher than the clipping level.

$$
g\left(x_{n}\right)= \begin{cases}x_{n} & \left|x_{n}\right| \leq \gamma \\ \gamma \mathrm{e}^{\mathrm{j} \phi\left(x_{n}\right)}, & \left|x_{n}\right|>\gamma\end{cases}
$$

Where $g\left(x_{n}\right)$ is the clipped output sequence, and $\phi\left(x_{n}\right)$ is the phase of $g\left(x_{n}\right)$ and $\gamma$ is the clipping amplitude .In clipping algorithm, clipping ratio (CR) is an important parameter which determines the value of $\gamma$. It is defined as

$$
C R=20 \log _{10} \frac{\gamma}{\sigma} \quad(\mathrm{dB})
$$

where $\sigma$ is the root mean squared value of the signal $x_{n}$ [7].

The higher the clipping ratio, few OFDM samples will be clipped. So it is important to choose the CR carefully to get a good reduction in the PAPR without degrade the performance of BER [8].

According to Eq.10, when clipping is performed to single signal point, we can get that the computational complexity includes 1 real addition, 4 real multiplications, 2 real divisions and 1 comparison. If OFDM signal samples with $\mathrm{N}$ sub-carriers are clipped by the clipping probability $\mathrm{p}$, the total computational complexity becomes $N p$ real addition, $4 N p$ real multiplication, $2 N p$ real division and $N p$ comparisons [7].

\section{SLM-CLIPPING}

SLM achieves good PAPR reduction performance at the cost of extra high computational complexity while clipping has moderate complexity but with nonlinear process and in-band distortion. To make a good balance between performance and complexity, a novel PAPR reduction technique is proposed based on combining SLM and clipping method. The diagram of the SLM-clip method is shown in Fig.3.

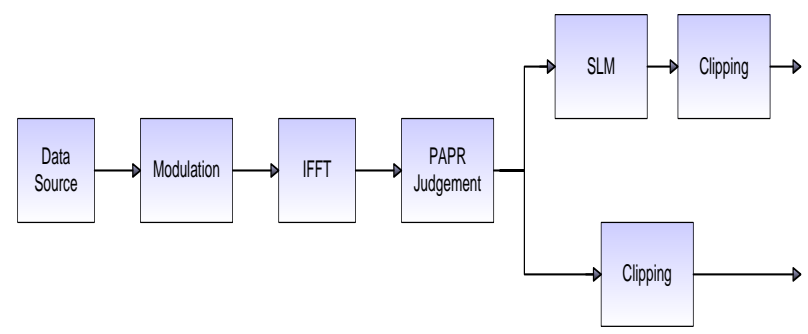

Fig.3 Block diagram of SLM and clipping method

Firstly, the PAPR of input OFDM signals are compared with a preset threshold $\gamma_{1}$, so as to judge whether the operation of SLM is needed. If the PAPR exceeds the preset threshold, SLM is performed. Then, clipping with $\gamma_{2}=$ $\mathrm{CR}$ is performed to the ultimate signal. The SLM-clip technique is summarized as follows.

1) Calculate the PAPR of the input OFDM signal.

2) Compare the PAPR with the preset threshold $\gamma_{1}$.

3 ) If the PAPR is smaller than $\gamma_{1}$, directly proceed to step 4 , else execute the SLM operation.

4) Executing the clipping operation with $\mathrm{CR} \gamma_{2}$ to control the PAPR of the ultimate signal.

\section{PTS-CLIPPING}

This method works same as SLM-clip technique as discuses above. Instead of SLM, PTS operation has to be performed. The diagram of the PTS-clip method is shown in Fig. 4. 


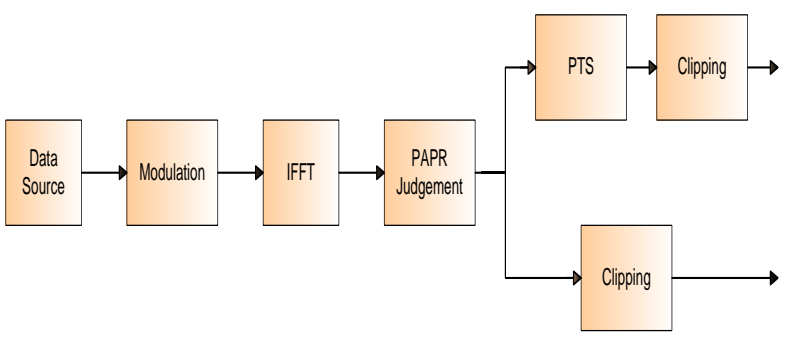

Fig. 4 Block diagram of PTS and clipping method

The PTS-clip technique is summarized as follows.

1) Calculate the PAPR of the input OFDM signal.

2) Compare the PAPR with the preset threshold. $\gamma_{1}$

3) If the PAPR is smaller than $\gamma_{1}$, directly proceed to step 4; else execute the PTS operation.

4) Executing the clipping operation with $\gamma_{2}=\mathrm{CR}$ to control the PAPR of the ultimate signal [7].

\section{DESIGN AND SIMULATION RESULT}

The OFDM system is implemented using MATLAB to allow various parameters of the system to be varied and tested. The following OFDM system parameters are considered.

Mapping: 16- QAM

Number of data sub-carriers: 52

Number of FFT points: 64

Channel Mode: AWGN

Data rate: $54 \mathrm{Mbps}$

Over sampling factor: 2

Total no. of symbols to TX. : 10000

The 64 point IFFT mapping is shown in Fig.5. For generation of real output, IFFT mapping is done by taking its conjugate.

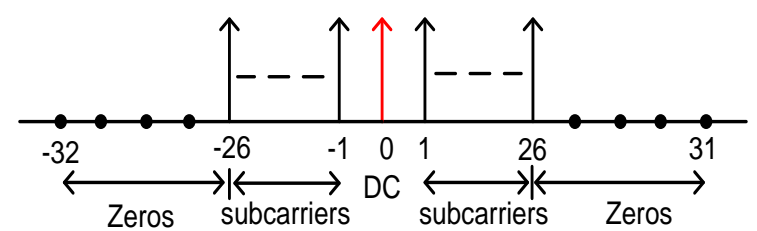

Fig. 5 IFFT mapping for complex time output OFDM signal

According to definition of clipping ratio given by Eq. 11, CR of 0.8 means the clipping level is about $3 \mathrm{~dB}$ lower than the rms level and a CR of $2 \mathrm{~dB}$ means the clipping level is about $3 \mathrm{~dB}$ higher than the rms level. Fig.6 shows that $\mathrm{CR}=0.8$ reduces $\mathrm{PAPR}$ much more compare to $\mathrm{CR}=2$ or 4.

Fig. 7 shows that BER is dependent on the clipping ratio (CR). Very less and very high values of clipping ratio is of no use, hence middle values are used. With increase in clipping the bit errors increases which degrades the BER performance. Signal can be purposely clipped by $3-4 \mathrm{~dB}$ so that the peak to average ratio can be reduced and BER performance closely matches with original OFDM transmission.

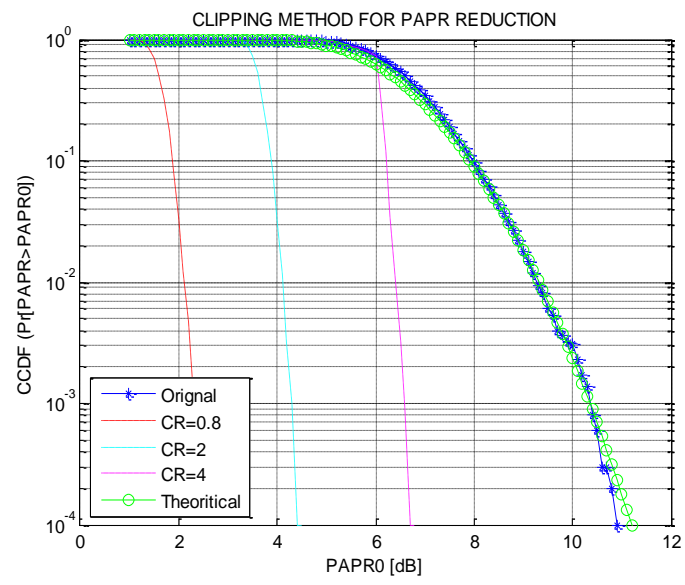

Fig. 6 CCDF plot for clipping method with different clipping ratio

BER performance is degraded due to heavy clipping with $\mathrm{CR}=0.8$.

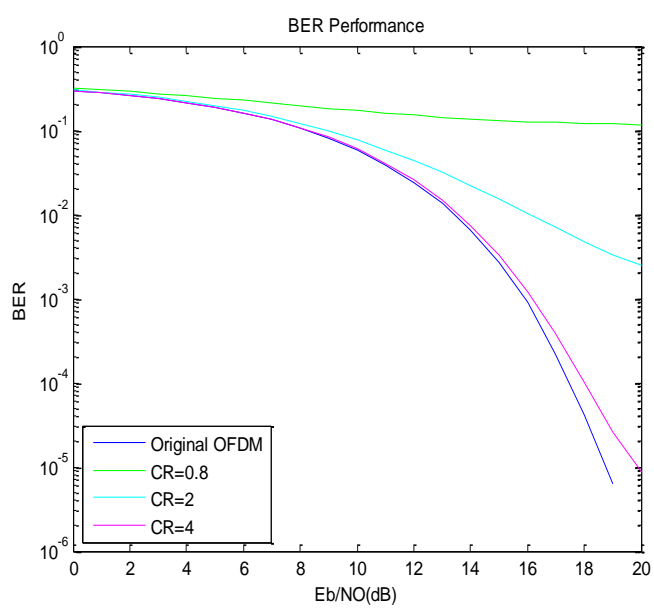

Fig 7. BER plot for clipping method with different ratio

Fig.8 shows the complementary cumulative distribution function (CCDF) of PAPR for the proposed technique, SLM and original OFDM. The SLM has $\mathrm{U}=4$ alternative signal representation (IFFTs).

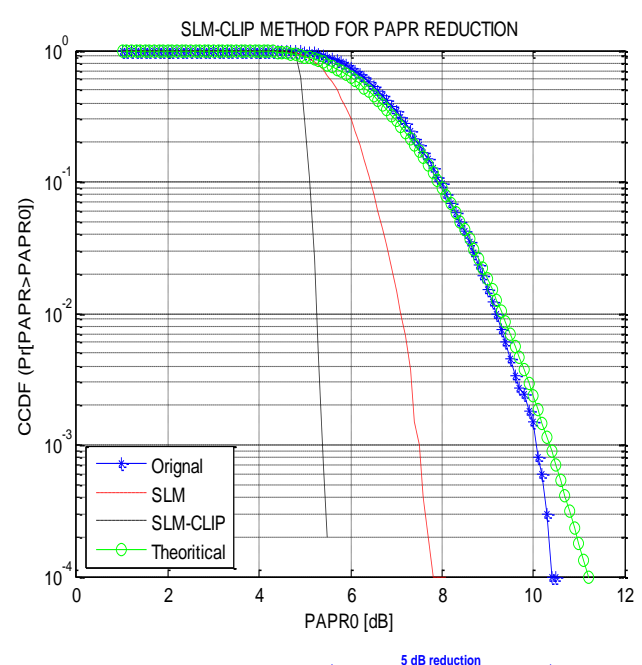

Fig. 8 CCDF plot for combined SLM-Clip method 
Because of the introduction of clipping, the PAPR reduction performance is improved much more than conventional SLM. In conventional SLM with U $=4$, PAPR reduction is of $3.2 \mathrm{~dB}$ whereas in SLM-Clip method, PAPR reduction is of $5 \mathrm{~dB}$. The performance and complexity of the proposed technique are dependent on the preset threshold and clipping ratio. Preset threshold of $8 \mathrm{~dB}$ and $\mathrm{CR}=4$ is use for simulation result.

From the result of simulation, the PAPR of original OFDM exceeding $8 \mathrm{~dB}$ is about $9-10 \%$ of the signals. Results for OFDM symbol are shown in Table I.

Table I Result of SLM-Clip method

\begin{tabular}{|c|c|c|c|}
\hline $\begin{array}{c}\text { OFDM } \\
\text { Symbol }\end{array}$ & $\begin{array}{c}\text { PAPR } \\
\text { Original }\end{array}$ & $\begin{array}{c}\text { SLM } \\
\text { U=4 }\end{array}$ & $\begin{array}{c}\text { SLM-Clip } \\
\text { CR=4 }\end{array}$ \\
\hline 1 & 6.1804 & 6.1804 & 4.9917 \\
\hline 10 & 8.8014 & 8.0554 & 5.9817 \\
\hline 100 & 6.7492 & 5.8743 & 4.8988 \\
\hline 500 & 5.2304 & 5.2304 & 4.7961 \\
\hline 1000 & 6.9845 & 5.1877 & 4.9566 \\
\hline 10000 & 6.5267 & 5.1524 & 4.8918 \\
\hline
\end{tabular}

Using the proposed method, the SLM computational complexity reduces to $9-10 \%$ of conventional SLM. The comparison of the complexity of SLM and the proposed method is shown in Table II.

Table II Complexity analysis of SLM-Clip method

\begin{tabular}{|l|c|c|}
\hline \multicolumn{1}{|c|}{ Operation } & $\begin{array}{c}\text { Complexity of } \\
\text { SLM }\end{array}$ & $\begin{array}{c}\text { Complexity } \\
\text { of SLM-Clip }\end{array}$ \\
\hline Complex addition & 1536 & 138.24 \\
\hline $\begin{array}{l}\text { Complex } \\
\text { multiplication }\end{array}$ & 1024 & 92.16 \\
\hline
\end{tabular}

Calculation: $N=2^{n} \quad \mathrm{~N}=64$ sub carrier, $\mathrm{U}=4$ In conventional SLM,

Complex addition $=\mathrm{NnU}$

$=64 * 6 * 4=1536$

Complex multiplication $=(\mathrm{N} / 2) n U+N U$

$$
=32 * 6 * 4+64 * 4
$$

$=1024$

In SLM-Clip,

Complex addition $=9 \%$ of conventional SLM

$$
=138.24
$$

Complex multiplication $=9 \%$ of conventional SLM

$$
=92.16
$$

By combining clipping method with PTS method, the PAPR reduction performance is improved much more than conventional PTS. Fig.9 shows the complementary cumulative distribution function (CCDF) of PAPR of the proposed technique, PTS and original OFDM. The PTS has V $=4$ sub-blocks and $\mathrm{W}=4$ allowed phase factors.

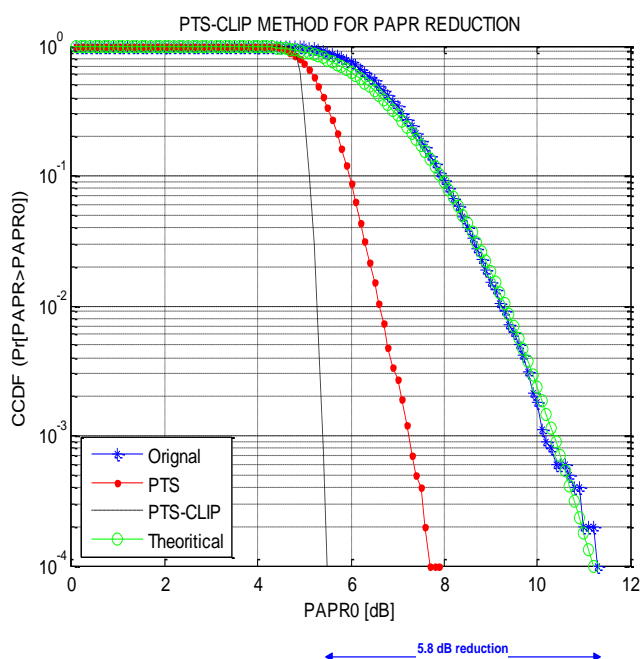

Fig. 9 CCDF plot for combined PTS-Clip method

Results for OFDM symbol are shown in Table III.

Table III Result of PTS-Clip method

\begin{tabular}{|c|c|c|c|}
\hline $\begin{array}{c}\text { OFDM } \\
\text { Symbol }\end{array}$ & $\begin{array}{c}\text { PAPR } \\
\text { Original }\end{array}$ & $\begin{array}{c}\text { PTS } \\
\text { W=4, V=4 }\end{array}$ & $\begin{array}{c}\text { PTS- Clip } \\
\text { CR=4 }\end{array}$ \\
\hline 1 & 5.7797 & 4.7833 & 4.8913 \\
\hline 10 & 5.8768 & 5.2475 & 4.8306 \\
\hline 100 & 6.0139 & 6.0370 & 4.8608 \\
\hline 500 & 7.7029 & 5.9282 & 5.1075 \\
\hline 1000 & 4.7130 & 4.8515 & 4.7130 \\
\hline 10000 & 8.0886 & 5.0808 & 4.8742 \\
\hline
\end{tabular}

In conventional PTS with $\mathrm{V}=4$ and $\mathrm{W}=4$, PAPR reduction is of $3.2 \mathrm{~dB}$ whereas in PTS-Clip method, PAPR reduction is of $5.8 \mathrm{~dB}$. The performance and complexity of the proposed technique are dependent on the preset threshold and clipping ratio. Preset threshold of $8 \mathrm{~dB}$ and $\mathrm{CR}=4$ is use for simulation result. From the result of simulation, the PAPR of original OFDM exceeding $8 \mathrm{~dB}$ is about $9-10 \%$ of the signals. Using the proposed method, the PTS computational complexity reduces to $9-10 \%$ of conventional PTS. The comparison of the complexity of PTS and the proposed method is shown in Table IV.

Table IV: Complexity analysis of PTS-Clip method

\begin{tabular}{|c|c|c|}
\hline Operation & $\begin{array}{c}\text { Complexity } \\
\text { of PTS }\end{array}$ & $\begin{array}{c}\text { Complexity of } \\
\text { PTS-Clip }\end{array}$ \\
\hline Complex addition & 13824 & 1244.2 \\
\hline $\begin{array}{l}\text { Complex multiplica- } \\
\text { tion }\end{array}$ & 21248 & 1912.3 \\
\hline Comparison & 4096 & 368.64 \\
\hline
\end{tabular}

Calculation: $N=2^{n} \mathrm{~N}=64$ sub carrier, $\mathrm{V}=4, \mathrm{~W}=4$ In conventional PTS,

Complex addition $=V \cdot\left(N \cdot \log _{2} N\right)+W^{V-1} \cdot N \cdot(V-1)$

$$
\begin{aligned}
& =4 * 64 * 6+64 * 64 * 3 \\
& =13824
\end{aligned}
$$

Complex multiplication $=$

$V \cdot\left((N / 2) \cdot \log _{2} N\right)+W^{V-1} \cdot N \cdot(V+1)$

$$
=4 * 32 * 6+64 * 64 * 5=21248
$$




$$
\begin{aligned}
\text { Complex comparison } & =W^{V-1} \cdot N \\
& =64 * 64=4096
\end{aligned}
$$

In PTS-Clip,

Complex addition $=9 \%$ of conventional PTS

$$
=1244.2
$$

Complex multiplication $=9 \%$ of conventional PTS

$$
=1912.3
$$

Complex comparison $=9 \%$ of conventional PTS

$$
=368.64
$$

\section{CONCLUSION}

Clipping is a simple, distortion method with minimal computational complexity. Signal can be purposely clipped with $\mathrm{CR}=4 \mathrm{~dB}$ so that the peak to average ratio can be reduced by $4.2 \mathrm{~dB}$ and BER performance closely matches with original OFDM transmission. By combining SLM-Clip method and PTS-Clip method, good balance between PAPR reduction performance and complexity can be achieved. SLM/PTS computational complexity reduces to $9-10 \%$ of conventional method. SLM-Clip can reduce 5 $\mathrm{dB}$ PAPR with $\mathrm{U}=4$ and $\mathrm{CR}=4$. PTS-Clip can reduce 5.8 dB PAPR with $\mathrm{V}=4, \mathrm{~W}=4$ and $\mathrm{CR}=4$.

\section{REFERENCES}

1) Carole Devlin, Anding Zhu, Thomas J. Brazil, "PAPR reduction technique for OFDM signals using unused tones with phase information," Circuits and Systems for Communications, 2008. ICCSC 2008. 4th IEEE International Conference, pp.6-10.

2) Stefan H. Muller , Johannes B. Huber., "A Comparison of peak power reduction schemes for OFDM," Proc. Of the IEEE Global Telecommunications Conference GLOBECOM'97, Phoenix, Arizona, USA, pp.1-5, November 1997.

3) Seok-Joong Heo,Hyung-Suk Noh, Jong-Seon No, Dong-Joon Shin., "A modified SLM scheme with low complexity for PAPR reduction of OFDM systems," The $18^{\text {th }}$ Annual IEEE International Symposiumon Personal, Indoor and Mobile Radio Communication(PIMRC'07)

4) S. Muller, R. Bauml, R. Fischer, and J. Huber, "OFDM with Reduced Peak-to-Average Power Ratio by Multiple Signal Representation", Annals of Telecommunications, Vol. 52, No.1-2,pp. 58-67, February 1997.

5) Van Eetvelt, P., Wade, G., Tomlinson, M., "Peak to average power reduction for OFDM schemes by selective scrambling," Electronics Letters, Vol.32,No.21,pp.1963-1964,1996.

6) Seung Hee Han, Jae Hong Lee, "An overview of peak-to-average power ratio reduction techniques for multicarrier transmission," IEEE wireless commun., Vol. 12, No. 2, pp. 56-65, April 2005.

7) Qing-Song Wen, Yue Xiao, Shao-Qian Li, H.Kayama, Chunlin Yan, "The implement of low-PAPR OFDM system," IEEE International Conference On Communications, Circuits and systems Proceddings, Vol. 4, pp. 1226-1229, June 2006

8) Ibrahim Ismail Al-kebsi, Mahamod Ismail, Kasmiran Jumari, T. A. Rahman, "Eliminate the effects of clipping technique on the SER performance by recovering the clipped part of the OFDM signal," International Journal of Computer Science and Network Security, Vol.9 No.7, July 2009. 